\title{
Three-dimensional symmetric shift radix systems
}

\author{
by
}

Andrea Huszti (Debrecen), Klaus Scheicher (Linz), Paul Surer (Leoben), and Jörg M. Thuswaldner (Leoben)

1. Introduction. In Akiyama et al. [1] a dynamical system called a shift radix system has been introduced.

Definition 1.1 (cf. [1]). Let $d \geq 1$ be an integer, $\mathbf{r} \in \mathbb{R}^{d}$, and let

$$
\widetilde{\tau}_{\mathbf{r}}: \mathbb{Z}^{d} \rightarrow \mathbb{Z}^{d}, \quad \mathbf{a}=\left(a_{1}, \ldots, a_{d}\right) \mapsto\left(a_{2}, \ldots, a_{d},-\lfloor\mathbf{r a}\rfloor\right),
$$

where $\mathbf{r a}=r_{1} a_{1}+\cdots+r_{d} a_{d}$, i.e., the inner product of the vectors $\mathbf{r}$ and $\mathbf{a}$. Then $\widetilde{\tau}_{\mathbf{r}}$ is called a shift radix system (SRS for short) if

$$
\forall \mathbf{a} \in \mathbb{Z}^{d} \exists n \in \mathbb{N}: \widetilde{\tau}_{\mathbf{r}}^{n}(\mathbf{a})=\mathbf{0} .
$$

SRS are related to number systems like $\beta$-expansions (cf. for instance $[8,9,11]$ ) or canonical number systems (cf. for instance [10]). Indeed, they form a unification and generalization of these notions of number systems. More details about SRS and their relation to $\beta$-expansions and canonical number systems can be found in $[1-3,13]$. In this paper we want to deal with a variant of SRS, the so-called symmetric shift radix systems.

Definition 1.2 (cf. [4]). Let $d \geq 1$ be an integer, $\mathbf{r} \in \mathbb{R}^{d}$, and let

$$
\tau_{\mathbf{r}}: \mathbb{Z}^{d} \rightarrow \mathbb{Z}^{d}, \quad \mathbf{a}=\left(a_{1}, \ldots, a_{d}\right) \mapsto\left(a_{2}, \ldots, a_{d},-\lfloor\mathbf{r a}+1 / 2\rfloor\right) .
$$

Then $\tau_{\mathbf{r}}$ is called a symmetric shift radix system (SSRS for short) if

$$
\forall \mathbf{a} \in \mathbb{Z}^{d} \exists n \in \mathbb{N}: \tau_{\mathbf{r}}^{n}(\mathbf{a})=\mathbf{0} .
$$

Observe that the only difference between the two definitions is the additional summand " $+1 / 2$ " inside the floor function in (1.1).

2000 Mathematics Subject Classification: Primary 11A63.

Key words and phrases: beta expansion, canonical number system, shift radix system.

The third and fourth authors are supported by the Austrian Research Foundation (FWF), Project S9610, that is part of the Austrian Research Network "Analytic Combinatorics and Probabilistic Number Theory".

All authors are supported by the "Aktion Österreich-Ungarn" project 63 öu3. 
SSRS have already been treated by Akiyama and Scheicher [4]. It was proved there that, analogously to the classical SRS, we have a strong relationship to certain notions of number systems. In particular SSRS form a common generalization of symmetric $\beta$-expansions and symmetric canonical number systems (SCNS). For completeness we recall the definition of these number systems and summarize the results on their relation to SSRS.

Definition 1.3 (cf. [4]). Let $\beta>1$ be a real non-integral number. The unique representation of a positive $\gamma \in \mathbb{R}$ of the form

$$
\gamma=d_{m} \beta^{m}+d_{m-1} \beta^{m-1}+d_{m-2} \beta^{m-2}+\cdots
$$

for some $m \in \mathbb{Z}$ with $d_{k} \in(-(\beta+1) / 2, \ldots,(\beta+1) / 2) \cap \mathbb{Z}, k \leq m$, such that the condition

$$
-\frac{\beta^{k+1}}{2} \leq \sum_{i \leq k} d_{i} \beta^{i}<\frac{\beta^{k+1}}{2}
$$

is satisfied for any $k \leq m$, is called the symmetric $\beta$-expansion of $\gamma$. We say that $\beta$ has property (SF) if all $\gamma \in \mathbb{Z}\left[\beta^{-1}\right]$ admit a finite symmetric $\beta$-expansion.

In the same way as for property (F) of ordinary $\beta$-expansions (see [8]) it can be shown that a number $\beta$ with property (SF) is necessarily a Pisot number.

Theorem 1.4 (cf. [4, Theorem 3.6]). A Pisot number $\beta$ with minimal polynomial $(x-\beta)\left(x^{d-1}+r_{d-1} x^{d-2}+\cdots+r_{2} x+r_{1}\right)$ has Property (SF) if and only if $\tau_{\left(r_{1}, \ldots, r_{d-1}\right)}$ is an SSRS.

There is a similar statement for SCNS whose definition we now recall.

Definition 1.5 (cf. [4]). Let $P(x)=x^{d}+a_{d-1} x^{d-1}+\cdots+a_{1} x+a_{0} \in \mathbb{Z}[x]$, $\left|a_{0}\right| \geq 2, \mathcal{R}:=\mathbb{Z}[x] / P(x) \mathbb{Z}[x], X \in \mathcal{R}$ the image of $x$ under the canonical epimorphism from $\mathbb{Z}[x]$ to $\mathcal{R}$ and $\mathcal{N}:=\left[-\left|a_{0}\right| / 2,\left|a_{0}\right| / 2\right) \cap \mathbb{Z} .(P(x), \mathcal{N})$ is called a symmetric canonical number system (SCNS) if each $R \in \mathcal{R}$ can be represented as

$$
R=\sum_{i=0}^{n} l_{i} X^{i}, \quad l_{i} \in \mathcal{N} .
$$

TheOREM 1.6 (cf. [4, Theorem 2.1]). $(P(x), \mathcal{N})$ with $P(x)=x^{d}+$ $a_{d-1} x^{d-1}+\cdots+a_{1} x+a_{0} \in \mathbb{Z}[x]$ and $\mathcal{N}:=\left[-\left|a_{0}\right| / 2,\left|a_{0}\right| / 2\right) \cap \mathbb{Z}$ is an $S C N S$ if and only if $\tau_{\mathbf{r}}$ is an SSRS, where $\mathbf{r}=\left(1 / a_{0}, a_{d-1} / a_{0}, \ldots, a_{1} / a_{0}\right)$.

Now, in order to show the differences between SSRS and SRS, we define the following sets related to the behavior of the orbits of $\widetilde{\tau}_{\mathbf{r}}$ and $\tau_{\mathbf{r}}$, 
respectively. Let

$$
\begin{aligned}
& \widetilde{\mathcal{D}}_{d}:=\left\{\mathbf{r} \in \mathbb{R}^{d} \mid \forall \mathbf{a} \in \mathbb{Z}^{d} \exists n, l \in \mathbb{N}: \widetilde{\tau}_{\mathbf{r}}^{k}(\mathbf{a})=\widetilde{\tau}_{\mathbf{r}}^{k+l}(\mathbf{a}) \forall k \geq n\right\}, \\
& \widetilde{\mathcal{D}}_{d}^{0}:=\left\{\mathbf{r} \in \mathbb{R}^{d} \mid \widetilde{\tau}_{\mathbf{r}} \text { is an } \operatorname{SRS}\right\},
\end{aligned}
$$

as well as

$$
\begin{aligned}
& \mathcal{D}_{d}:=\left\{\mathbf{r} \in \mathbb{R}^{d} \mid \forall \mathbf{a} \in \mathbb{Z}^{d} \exists n, l \in \mathbb{N}: \tau_{\mathbf{r}}^{k}(\mathbf{a})=\tau_{\mathbf{r}}^{k+l}(\mathbf{a}) \forall k \geq n\right\} \\
& \mathcal{D}_{d}^{0}:=\left\{\mathbf{r} \in \mathbb{R}^{d} \mid \tau_{\mathbf{r}} \text { is an SSRS }\right\} .
\end{aligned}
$$

For $\mathbf{r}=\left(r_{1}, \ldots, r_{d}\right) \in \mathbb{R}^{d}$, let

$$
R(\mathbf{r})=\left(\begin{array}{ccccc}
0 & 1 & 0 & \cdots & 0 \\
\vdots & 0 & \ddots & \ddots & \vdots \\
\vdots & \vdots & \ddots & 1 & 0 \\
0 & 0 & \cdots & 0 & 1 \\
-r_{1} & -r_{2} & \cdots & -r_{d-1} & -r_{d}
\end{array}\right)
$$

For $M \in \mathbb{R}^{d \times d}$, denote by $\varrho(M)$ the spectral radius of $M$, i.e., the maximum absolute value of the eigenvalues of $M$. For simplicity, we write $\varrho(\mathbf{r}):=$ $\varrho(R(\mathbf{r}))$. Let

$$
\mathcal{E}_{d}(\varepsilon)=\left\{\mathbf{r} \in \mathbb{R}^{d}: \varrho(\mathbf{r})<\varepsilon\right\} .
$$

It is known that $\overline{\mathcal{E}_{d}(\varepsilon)}$ is a regular set, i.e., it coincides with the closure of its interior.

We start by comparing the sets $\mathcal{D}_{d}$ and $\widetilde{\mathcal{D}}_{d}$. Firstly, it can easily be seen that their interiors are the same since from [1] we know that $\mathcal{E}_{d}(1) \subset \widetilde{\mathcal{D}}_{d} \subset$ $\overline{\mathcal{E}_{d}(1)}$ while in [4] it has been shown that

$$
\mathcal{E}_{d}(1) \subset \mathcal{D}_{d} \subset \overline{\mathcal{E}_{d}(1)}
$$

We will dwell upon the set $\mathcal{D}_{d}$ in Section 2 . However, the sets $\mathcal{D}_{d}^{0}$ and $\widetilde{\mathcal{D}}_{d}^{0}$ have different behavior. The properties of $\widetilde{\mathcal{D}}_{d}^{0}$ have been studied in [1-3]. In $[2,13]$ special attention was paid to the two-dimensional case $\widetilde{\mathcal{D}}_{2}^{0}$. It turns out that the structure of $\widetilde{\mathcal{D}}_{2}^{0}$ is very complicated and although large parts of the set could be characterized, a full characterization is still outstanding. An approximation of $\widetilde{\mathcal{D}}_{2}^{0}$ is shown in Figure 1 .

The sets $\widetilde{\mathcal{D}}_{d}^{0}$ for $d \geq 3$ are not yet investigated in detail; however, computer experiments indicate that $\widetilde{\mathcal{D}}_{3}^{0}$ is hard to describe.

For the case of SSRS the situation becomes more pleasant at least for low dimensions. Akiyama and Scheicher [4] presented the surprising result that $\mathcal{D}_{2}^{0}$ has a really simple characterization (see Figure 2). They found out 


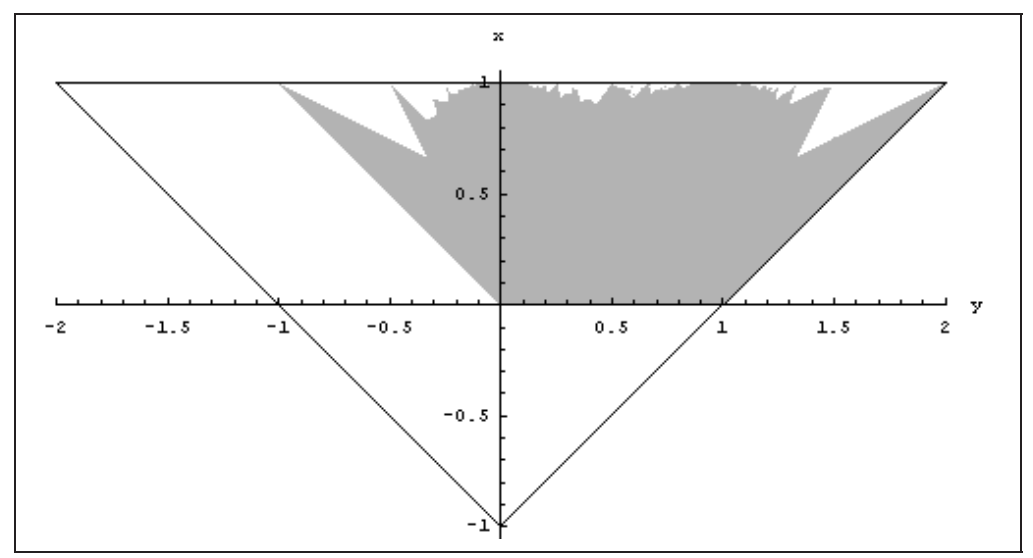

Fig. 1. An approximation of $\widetilde{\mathcal{D}}_{2}^{0}$

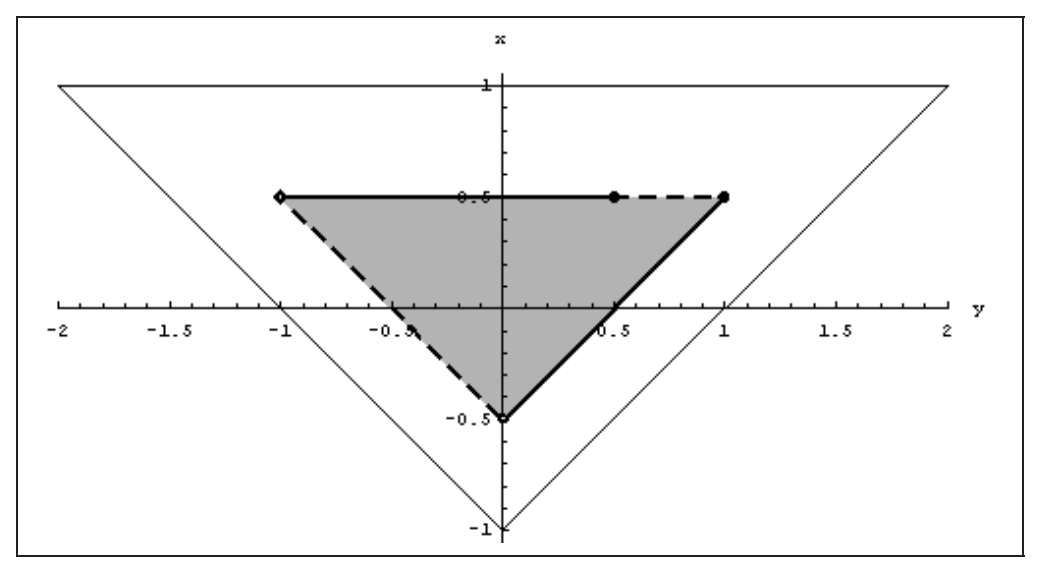

Fig. 2. The shape of $\mathcal{D}_{2}^{0}$

that

$$
\begin{aligned}
\mathcal{D}_{2}^{0}= & \left\{(x, y) \in \mathbb{R}^{2} \mid x \leq 1 / 2,-x-1 / 2<y \leq x+1 / 2\right\} \\
& \backslash\left\{(1 / 2, y) \in \mathbb{R}^{2} \mid 1 / 2<y<1\right\},
\end{aligned}
$$

i.e., $\mathcal{D}_{2}^{0}$ is an isosceles triangle together with some parts of its boundary. In the present paper we are interested in the shape of the set $\mathcal{D}_{3}^{0}$. Amazingly, we will see that $\mathcal{D}_{3}^{0}$ can be completely described as a simple as well as interesting body.

The paper is organized as follows. In Section 2 we concentrate on $\mathcal{D}_{d}$ and its relation to $\mathcal{D}_{d}^{0}$ in general and specially if $d=3$. Furthermore, we present an algorithm that is useful for the description of $\mathcal{D}_{d}^{0}$. It was first presented in [6] and later adapted for SSRS in [4]. In Section 3 we will state the exact characterization of the set $\mathcal{D}_{3}^{0}$. In Section 4 we will prove this characterization result by using the algorithm presented in Section 2 together with some other 
algorithms relating to bodies defined by polynomial inequalities such as the cylindrical algebraic decomposition algorithm (cf. Collins [7]).

2. Construction of $\mathcal{D}_{3}^{0}$ from $\mathcal{D}_{3}$. Let us consider the set $\mathcal{D}_{d}$. By (1.2), apart from the boundary, $\mathcal{D}_{d}$ coincides with $\mathcal{E}_{d}(1)$ and their closures are equal. As the minimal polynomial of $R(\mathbf{r})$ is

$$
x^{d}+r_{d} x^{d-1}+\cdots+r_{2} x+r_{1}
$$

the problem of characterizing $\mathcal{E}_{d}(\varepsilon)$ is equivalent to finding polynomials of the form (2.1) whose roots lie inside the $\varepsilon$ multiple of the unit ball. This problem was already settled in $[12,15]$. From these references we easily get the following lemma.

LEMMA 2.1. A vector $\mathbf{r}=\left(r_{1}, \ldots, r_{d}\right)$ is in $\mathcal{E}_{d}(\varepsilon)$ if and only if the Hermitian form

$$
H_{d}\left(x_{0}, \ldots, x_{d-1}\right):=\sum_{i=0}^{d-1}\left|\sum_{j=i}^{d-1} \varepsilon^{d+i-j} r_{d+i-j+1} x_{j}\right|^{2}-\sum_{i=0}^{d-1}\left|\sum_{j=i}^{d-1} \varepsilon^{j-i} r_{j-i+1} x_{j}\right|^{2}
$$

with $r_{d+1}=1$ is positive definite.

Now we turn to the study of $\mathcal{D}_{d}^{0}$. For this matter we recall the following definitions (cf. for instance Barnsley [5, Chapter IV, Definitions 3.1 and 3.2]).

Definition 2.2. Let $\tau_{\mathbf{r}}$ with $\mathbf{r} \in \mathbb{R}^{d}$ be given.

- Let $\mathbf{x} \in \mathbb{Z}^{d}$. Then the set $\left\{\tau_{\mathbf{r}}^{n}(\mathbf{x}) \mid n \in\{0,1,2, \ldots\}\right\}$ is called the orbit of $\mathbf{x}$.

- A point $\mathbf{x} \in \mathbb{Z}^{d}$ is called a periodic point if there is a positive integer $L$ such that $\mathbf{x}=\tau_{\mathbf{r}}^{L}(\mathbf{x})$. The integer $L$ is called a period of $\mathbf{x}$.

- The orbit of a periodic point is called a cycle.

The set $\mathcal{D}_{d}^{0}$ can be constructed from $\mathcal{D}_{d}$ by cutting out convex polyhedra. For $\mathbf{r}=\left(r_{1}, \ldots, r_{d}\right) \in \mathcal{D}_{d}$ an element $\mathbf{a}=\left(a_{1}, \ldots, a_{d}\right) \in \mathbb{Z}^{d} \backslash\{0\}$ is a non-zero periodic point of $\tau_{\mathbf{r}}$ of period $L$ if $\mathbf{a}=\tau_{\mathbf{r}}^{L}(\mathbf{a})$. From the definition of $\mathcal{D}_{d}^{0}$ it follows that the existence of such a periodic point is necessary and sufficient for $\mathbf{r} \notin \mathcal{D}_{d}^{0}$. Suppose that the orbit of a (which is in fact a cycle) consists of the points

$$
\tau_{\mathbf{r}}^{j}(\mathbf{a})=\left(a_{1+j}, \ldots, a_{d+j}\right) \quad(0 \leq j \leq L-1)
$$

where $a_{L+1}=a_{1}, \ldots, a_{L+d-1}=a_{d-1}$. We denote such a cycle by

$$
\left(a_{1}, \ldots, a_{d}\right) ; a_{d+1}, \ldots, a_{L}
$$

and say that it is a cycle of $\tau_{\mathbf{r}}$ or just a cycle of $\mathcal{D}_{d}$.

Let a non-zero cycle $\pi:=\left(a_{1}, \ldots, a_{d}\right) ; a_{d+1}, \ldots, a_{L}$ be given. We may ask for the set $P(\pi)$ of all $\mathbf{r} \in \mathcal{D}_{d}$ for that $\pi$ occurs as a cycle of $\tau_{\mathbf{r}}$. By the 
definition of $\tau_{\mathbf{r}}$, an element $\mathbf{r} \in P(\pi)$ has to satisfy the system of $L$ double inequalities

$$
-1 / 2 \leq r_{1} a_{1+i}+r_{2} a_{2+i}+\cdots+r_{d} a_{d+i}+a_{d+1+i}<1 / 2 .
$$

Here $i$ runs from 0 to $L-1$ and $a_{L+1}=a_{1}, \ldots, a_{L+d}=a_{d}$. Such a system characterizes a convex polyhedron, which is possibly degenerate or empty. Therefore we will call $P(\pi)$ a cutout polyhedron. Example 2.5 shows what $P(\pi)$ could look like for a given cycle in the three-dimensional case. Since each point $\mathbf{r} \in P(\pi)$ has $\pi$ as a cycle of the associated mapping $\tau_{\mathbf{r}}$, the set $P(\pi)$ has empty intersection with $\mathcal{D}_{d}^{0}$. Thus we get the representation

$$
\mathcal{D}_{d}^{0}=\mathcal{D}_{d} \backslash \bigcup_{\pi \neq 0} P(\pi),
$$

where the union is extended over all non-zero cycles $\pi$. Since the set of cycles can a priori be infinite, this expression is not suitable for calculations. The following theorem shows how to reduce the set of possible cycles to a finite set and gives an efficient algorithm for a closed subset $H$ of int $\mathcal{D}_{d}=\mathcal{E}_{d}(1)$ to determine $H \cap \mathcal{D}_{d}^{0}$. It was presented for the first time for canonical number systems in [6] and was further improved and adapted to SRS in $[1,2,13]$. In [4] the algorithm was established for SSRS. Basically we will use this version. Let $\mathbf{e}_{i}$ be the $i$ th canonical unit vector. For an $\mathbf{r}=\left(r_{1}, \ldots, r_{d}\right) \in \operatorname{int} \mathcal{D}_{d}$, denote by $\mathcal{V}(\mathbf{r}) \subset \mathbb{Z}^{d}$ the smallest set with the properties

(1) $\pm \mathbf{e}_{i} \in \mathcal{V}(\mathbf{r}), i=1, \ldots, d$,

(2) $\left(a_{1}, \ldots, a_{d}\right) \in \mathcal{V}(\mathbf{r}) \Rightarrow\left(a_{2}, \ldots, a_{d+1}\right) \in \mathcal{V}(\mathbf{r})$ where $a_{d+1}$ satisfies

$$
-1<r_{1} a_{1}+\cdots+r_{d} a_{d}+a_{d+1}<1 \text {. }
$$

$\mathcal{V}(\mathbf{r})$ is called a set of witnesses for $\mathbf{r}$. Additionally define $\mathcal{G}(\mathcal{V}(\mathbf{r}))=V \times E$ to be the graph with set of vertices $V=\mathcal{V}(\mathbf{r})$ and set of edges $E \subset V \times V$ such that

$$
\forall \mathbf{a} \in V:\left(\mathbf{a}, \tau_{\mathbf{r}}(\mathbf{a})\right) \in E .
$$

The set of vertices is exactly the same as in [1]. The edges are defined in a different way. There exists only one outgoing edge for each vertex. We are interested in the cyclic structure of such graphs. A cycle $\mathbf{a}_{1} \rightarrow \mathbf{a}_{2} \rightarrow \cdots \rightarrow$ $\mathbf{a}_{L} \rightarrow \mathbf{a}_{1}$ in the graph $\mathcal{G}(\mathcal{V}(\mathbf{r}))$ induces a periodic point of period $L$ (and therefore a cycle) for $\tau_{\mathbf{r}}$ in an obvious way.

Theorem 2.3 (cf. [4, Theorem 4.2]). Let $\mathbf{r}_{1}, \ldots, \mathbf{r}_{k} \in \mathcal{D}_{d}$ and let $H:=$ $\square\left(\mathbf{r}_{1}, \ldots, \mathbf{r}_{k}\right)$ be the convex hull of $\mathbf{r}_{1}, \ldots, \mathbf{r}_{k}$. Assume that $H \subset \operatorname{int} \mathcal{D}_{d}$ and is sufficiently small in diameter. Then there exists an algorithm to construct a finite directed graph $G(H)=V \times E$ with vertices $V \subset \mathbb{Z}^{d}$ and edges $E \subset V \times V$ which satisfies

(1) $\pm \mathbf{e}_{i} \in V$ for all $i=1, \ldots, d$, 
(2) $\mathcal{G}(\mathcal{V}(\mathbf{x}))$ is a subgraph of $G(H)$ for all $\mathbf{x} \in H$,

(3) $H \cap \mathcal{D}_{d}^{0}=H \backslash \bigcup_{\pi} P(\pi)$, where $\pi$ runs through all cycles induced by the non-zero primitive cycles of $G(H)$.

REMARK 2.4. Note that there are cycles in the graph $G(H)$ that do not correspond to a cycle of any $\tau_{\mathbf{r}}$. In this case we set $P(\pi)=\emptyset$ because the set of inequalities in (2.2) has no solution.

Observe that the theorem can be extended to any convex set $H \subset \operatorname{int} \mathcal{D}_{d}$ analogously to [13]. In our context the version presented in Theorem 2.3 suffices. In practice, the graph in Theorem 2.3 is constructed by successively adding new vertices. Note that the "sufficiently small" restriction is essential. It turns out that the size of the set of vertices in the graph in Theorem 2.3 can grow to infinity if $H$ is chosen too large. For more details, see $[4,13]$. For us it is only important to choose $H$ in such a way that everything stays finite. This can be realized by a suitable subdivision of a given set. We will return to this problem in Section 4.

Theorem 2.3 proved to be a powerful tool for characterizing $\mathcal{D}_{d}^{0}$. If it is used properly, $\mathcal{D}_{d}^{0} \cap H$ can be characterized for any closed $H \subset \operatorname{int} \mathcal{D}_{d}$. Thus, whenever there exists such an $H$ with $\mathcal{D}_{d}^{0} \subset H$ there is a chance to characterize $\mathcal{D}_{d}^{0}$ completely. That was the case for $d=2$ and we will see that this is also valid for $d=3$. For classical SRS, there does not exist such a set $H$ for $d \geq 2$.

Our aim is to characterize $\mathcal{D}_{3}^{0}$. We already know that

$$
\mathcal{E}_{3}(1) \subset \mathcal{D}_{3} \subset \overline{\mathcal{E}_{3}(1)} \text {. }
$$

From Lemma 2.1 we calculate

$$
\mathcal{E}_{3}(1)=\left\{(x, y, z) \in \mathbb{R}^{3}|| x|<1,| y-x z\left|<1-x^{2},\right| x+z|<| y+1 \mid\right\} .
$$

The following example shows how a given cycle cuts out a polyhedron from $\mathcal{E}_{3}(1)$.

Example 2.5. Consider the cycle $\pi:=(1,1,-1) ;-1,0$. It induces a system of inequalities (2.2) which describes the polyhedron $P(\pi)$. In our case we get

$$
\begin{aligned}
P(\pi)=\{(x, y, z) \mid & -1 / 2 \leq x+y-z-1<1 / 2 \wedge-1 / 2 \leq x-y-z<1 / 2 \\
& \wedge-1 / 2 \leq-x-y+1<1 / 2 \wedge-1 / 2 \leq-x+z+1<1 / 2 \\
& \wedge-1 / 2 \leq y+z-1<1 / 2\} .
\end{aligned}
$$

By removing redundant inequalities, this reduces to

$$
\begin{array}{r}
P(\pi)=\{(x, y, z) \mid x+y-z-1<1 / 2 \wedge x-y-z<1 / 2 \wedge-1 / 2 \leq-x-y+1 \\
\wedge-x+z+1<1 / 2 \wedge-1 / 2 \leq y+z-1\}
\end{array}
$$

yielding a polyhedron with five faces. $P(\pi)$ only contains $\mathbf{r} \in \mathcal{D}_{d}$ with $\tau_{\mathbf{r}}^{5}((1,1,-1))=(1,1,-1)$, and hence $P(\pi)$ has empty intersection with $\mathcal{D}_{3}^{0}$. 


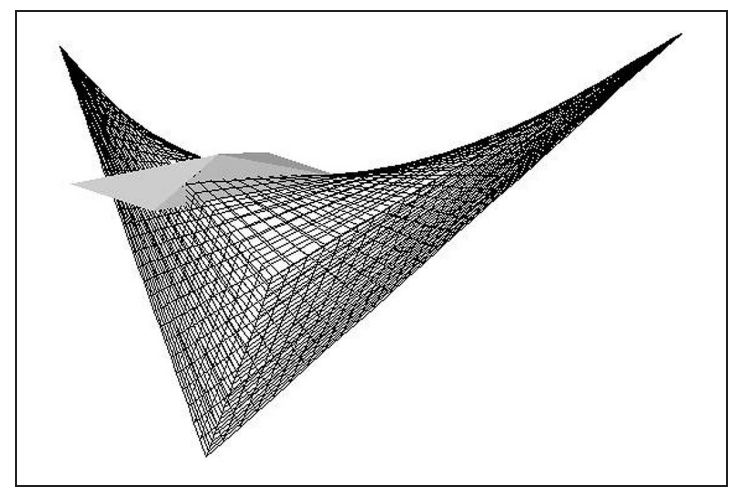

Fig. 3. The position of $P(\pi)$ in $\mathcal{E}_{3}(1)$

Figure 3 shows the position of $P(\pi)$ in $\mathcal{E}_{3}(1)$. It is easy to see that $P(\pi)$ really cuts out some part of $\mathcal{D}_{3}$.

Later on we will need $\overline{\mathcal{E}_{3}(1)}$ and there some problems occur. Consider the set which is obtained by changing all the strict inequalities (" $<$ ") in $(2.3)$ to non-strict inequalities (" $\leq$ "). One may think that it equals $\overline{\mathcal{E}_{3}(1)}$, but this is not the case. It can be easily seen that this set contains the unbounded $\underline{\text { lines }}(1, \lambda, \lambda), \underline{\lambda \in \mathbb{R}}$, and $(-1, \mu,-\mu), \mu \in \mathbb{R}$, which cannot be true for $\overline{\mathcal{E}_{3}(1)}$. Hence, $\overline{\mathcal{E}_{3}(1)}$ is only a subset of this set. We will solve the problem by adding some suitable inequalities. Let

$$
\begin{aligned}
\mathcal{E}_{3}^{\prime}:=\left\{(x, y, z) \in \mathbb{R}^{3}|| x \mid \leq\right. & 1 \wedge|y-x z| \leq 1-x^{2} \\
& \wedge|x+z| \leq|y+1| \wedge|y-1| \leq 2 \wedge|z| \leq 3\}
\end{aligned}
$$

and consider the intersection of $\mathcal{E}_{3}^{\prime}$ with the hyperplane

$$
A_{c}:=\left\{(x, y, z) \in \mathbb{R}^{3} \mid x-c=0\right\}
$$

for constant $c$.

Lemma 2.6. For any $c$ with $|c|<1$ the intersection of $\mathcal{E}_{3}^{\prime}$ with the plane $A_{c}$ is the closed triangle $\triangle\left(A_{c}^{(1)}, A_{c}^{(2)}, A_{c}^{(3)}\right)$ with $A_{c}^{(1)}=(c,-1,-c), A_{c}^{(2)}=$ $(c, 1-2 c, c-2), A_{c}^{(3)}=(c, 2 c+1, c+2)$.

Proof. We have

$$
\begin{array}{r}
\mathcal{E}_{3}^{\prime} \cap A_{c}=\left\{(c, y, z) \in \mathbb{R}^{3}|| y-c z\left|\leq 1-c^{2} \wedge\right| c+z|\leq| y+1 \mid\right. \\
\wedge|y-1| \leq 2 \wedge|z| \leq 3\} .
\end{array}
$$

As all inequalities are linear, this is a convex set. It is quickly verified that $A_{c}^{(1)}, A_{c}^{(2)}, A_{c}^{(3)} \in \mathcal{E}_{3}^{\prime} \cap A_{c}$. Thus $\triangle\left(A_{c}^{(1)}, A_{c}^{(2)}, A_{c}^{(3)}\right) \subset \mathcal{E}_{3}^{\prime} \cap A_{c}$. On the other hand, consider the closed convex set

$$
B_{c}:=\left\{(c, y, z) \mid y-c z \leq 1-c^{2} \wedge c+z \leq y+1 \wedge-y-1 \leq c+z\right\} .
$$


Observe that for its definition we used only inequalities that occurred in the definition of $\mathcal{E}_{3}^{\prime} \cap A_{c}$ and hence we have $\mathcal{E}_{3}^{\prime} \cap A_{c} \subset B_{c}$. Pairwise intersection of the three boundary lines of $B_{c}$ yields exactly the three points $A_{c}^{(1)}, A_{c}^{(2)}, A_{c}^{(3)}$ and therefore $\triangle\left(A_{c}^{(1)}, A_{c}^{(2)}, A_{c}^{(3)}\right)=B_{c} \supset \mathcal{E}_{3}^{\prime} \cap A_{c}$.

TheOREM 2.7. $\overline{\mathcal{E}_{3}(1)}=\mathcal{E}_{3}^{\prime}$.

Proof. Obviously $\mathcal{E}_{3}^{\prime}$ is a closed set while $\mathcal{E}_{3}(1)$ is open. We show that int $\mathcal{E}_{3}^{\prime}=\mathcal{E}_{3}(1)$. From Lemma 2.6 we know

$$
\mathcal{E}_{3}^{\prime} \cap A_{c}=\left\{(c, y, z) \mid y-c z \leq 1-c^{2} \wedge c+z \leq y+1 \wedge-y-1 \leq c+z\right\}
$$

and as every point of $\mathcal{E}_{3}(1)$ is inside $\mathcal{E}_{3}^{\prime} \cap A_{c}$ for some $|c|<1$ we have

$$
\mathcal{E}_{3}^{\prime}=\bigcup_{|c| \leq 1}\left(\mathcal{E}_{3}^{\prime} \cap A_{c}\right) \supset \mathcal{E}_{3}(1)
$$

and therefore

$$
\operatorname{int} \mathcal{E}_{3}^{\prime} \supset \operatorname{int} \mathcal{E}_{3}(1)=\mathcal{E}_{3}(1) .
$$

On the other hand, denote by $\operatorname{int}_{A_{c}}\left(\mathcal{E}_{3}^{\prime} \cap A_{c}\right)$ the interior of the set $\mathcal{E}_{3}^{\prime} \cap A_{c}$ (subspace topology) for $|c|<1$, i.e., the open triangle defined in Lemma 2.6, and observe that

$$
\operatorname{int} \mathcal{E}_{3}^{\prime}=\bigcup_{|c|<1} \operatorname{int}_{A_{c}}\left(\mathcal{E}_{3}^{\prime} \cap A_{c}\right)
$$

as we can find a neighborhood around each point of $\operatorname{int}_{A_{c}}\left(\mathcal{E}_{3}^{\prime} \cap A_{c}\right),|c|<1$, which is contained in $\mathcal{E}_{3}^{\prime}$. Further each point of $\operatorname{int}_{A_{c}}\left(\mathcal{E}_{3}^{\prime} \cap A_{c}\right)$ satisfies the conditions of $\mathcal{E}_{3}(1)$ whenever $|c|<1$. Hence

$$
\operatorname{int} \mathcal{E}_{3}^{\prime}=\bigcup_{|c|<1} \operatorname{int}\left(\mathcal{E}_{3}^{\prime} \cap A_{c}\right) \subset \mathcal{E}_{3}(1)
$$

Thus we have shown that int $\mathcal{E}_{3}^{\prime}=\mathcal{E}_{3}(1)$.

To prove the theorem we show $\mathcal{E}_{3}^{\prime}=\overline{\operatorname{int} \mathcal{E}_{3}^{\prime}}$. We already know that int $\mathcal{E}_{3}^{\prime}=$ $\bigcup_{|c|<1} \operatorname{int}_{A_{c}}\left(\mathcal{E}_{3}^{\prime} \cap A_{c}\right)$. Hence we look at the convergent sequences of points contained in $\bigcup_{|c|<1} \operatorname{int}\left(\mathcal{E}_{3}^{\prime} \cap A_{c}\right)$. Such a sequence converges either to some point in $\bigcup_{|c|<1}\left(\mathcal{E}_{3}^{\prime} \cap A_{c}\right)$ or to some point in one of the sets $\lim _{c \rightarrow \pm 1}\left(\mathcal{E}_{3}^{\prime} \cap A_{c}\right)$. From Lemma 2.6 we already have

$$
\mathcal{E}_{3}^{\prime} \cap A_{c}=\triangle((c,-1,-c),(c, 1-2 c, c-2),(c, 2 c+1, c+2))
$$

and we see that

$$
\begin{aligned}
\lim _{c \rightarrow 1}\left(\mathcal{E}_{3}^{\prime} \cap A_{c}\right) & =\{(1, \lambda, \lambda) \mid-1 \leq \lambda \leq 3\}, \\
\lim _{c \rightarrow-1}\left(\mathcal{E}_{3}^{\prime} \cap A_{c}\right) & =\{(-1, \lambda,-\lambda) \mid-1 \leq \lambda \leq 3\}
\end{aligned}
$$


which exactly correspond to the sets $\mathcal{E}_{3}^{\prime} \cap A_{ \pm 1}$. Thus

$$
\overline{\mathcal{E}_{3}(1)}=\overline{\operatorname{int} \mathcal{E}_{3}^{\prime}}=\bigcup_{|c| \leq 1}\left(\mathcal{E}_{3}^{\prime} \cap A_{c}\right)=\mathcal{E}_{3}^{\prime}
$$

and we are done.

Finally, we have a representation of the closure of $\mathcal{E}_{3}(1)$. In the proof of Lemma 2.6 we already recognized that the number of inequalities to describe $\overline{\mathcal{E}_{3}^{\prime}}$ can be reduced. Indeed, by using an algorithm (Algorithm 3) which we will present in Section 4, we gain

$$
\overline{\mathcal{E}_{3}(1)}=\left\{(x, y, z)|| x+z\left|\leq 1+y \wedge y-x z \leq 1-x^{2} \wedge\right| z \mid \leq 3\right\} .
$$

3. Statement of the main result. In this section we give a complete description of $\mathcal{D}_{3}^{0}$. To this end we define the sets

$$
\begin{aligned}
& S_{1}:=\{(x, y, z) \mid 2 x-2 z \geq 1 \wedge 2 x+2 y+2 z>-1 \wedge 2 x+2 y \leq 1 \\
& \wedge 2 x \leq 1 \wedge 2 x-2 y+2 z \leq 1\} \\
& S_{2}:=\{(x, y, z) \mid x-z \leq-1 \wedge 2 x-2 y+2 z \leq 1 \wedge-2 x+2 y \leq 1 \\
& \wedge 2 x>-1\} \\
& S_{3}:=\{(x, y, z) \mid x-z>-1 \wedge 2 x-2 y+2 z \leq 1 \wedge-2 x+2 y<1,2 x>-1 \\
& \wedge 2 x-2 z<-1 \wedge 2 x+2 y+2 z>-1\}, \\
& S_{4}:=\{(x, y, z) \mid 2 x-2 y+2 z \leq 1 \wedge-2 x+2 y \leq 1 \wedge 2 x-2 z=-1 \\
& \wedge 2 x+2 y+2 z>-1\}, \\
& S_{5}:=\{(x, y, z) \mid-1<2 x \leq 1 \wedge-1<2 x-2 z \leq 1 \wedge 2 x+2 y+2 z>-1 \\
& \wedge 2 x-2 y+2 z \leq 1 \wedge 2 x+4 y-2 z<3 \wedge 2 y \leq 1\}
\end{aligned}
$$

and denote their union by

$$
\mathcal{S}:=\bigcup_{i \in\{1, \ldots, 5\}} S_{i}
$$

Note that $S_{1}, S_{2}, S_{3}, S_{5}$ are polyhedra while $S_{4}$ is a polygon. The following theorem states the main result of the present paper.

Theorem 3.1. $\mathcal{D}_{3}^{0}=\mathcal{S}$.

Two views of the set $\mathcal{D}_{3}^{0}$ are depicted in Figures 4 and 5. For rotating $3 \mathrm{D}$-pictures of $\mathcal{D}_{3}^{0}$ we refer the reader to the authors' home pages [14].

We will prove this theorem in Section 4. Here we want to give an outline of the proof. In a first step we will use Theorem 2.3 to show that

$$
\mathcal{S} \subseteq \mathcal{D}_{3}^{0} \text {. }
$$

For the opposite inclusion we need a set $\Pi$ of non-zero cycles such that for $\mathcal{P}:=\bigcup_{\pi \in \Pi} P(\pi)$ we have

$$
\mathcal{S} \cup \mathcal{P} \supseteq \mathcal{D}_{3} .
$$




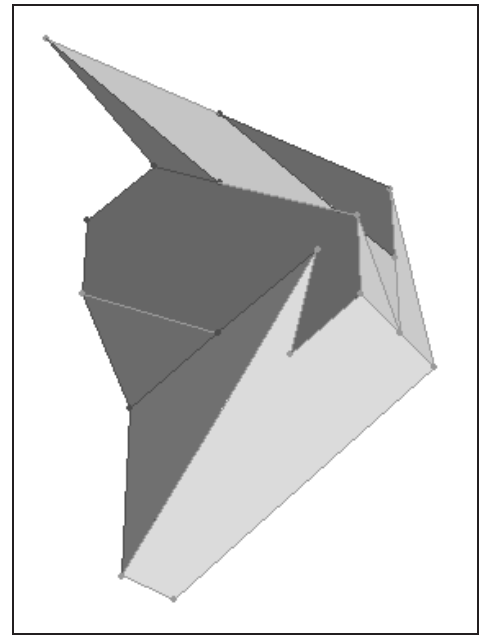

Fig. 4. A view of $\mathcal{D}_{3}^{0}$

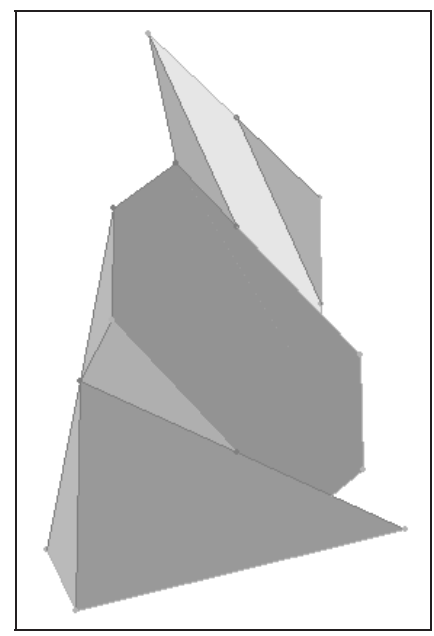

Fig. 5. A view of $\mathcal{D}_{3}^{0}$

From (3.1) we can deduce $\mathcal{S} \cap \mathcal{P}=\emptyset$. Thus,

$$
\mathcal{S} \supseteq \mathcal{D}_{3} \backslash \mathcal{P} \supseteq \mathcal{D}_{3}^{0}
$$

Since $\mathcal{D}_{3} \subset \overline{\mathcal{E}_{3}(1)}$ we are done if we can cover $\overline{\mathcal{E}_{3}(1)}$ with $\mathcal{P} \cup \mathcal{S}$, i.e., if we can show that

$$
\mathcal{P} \cup \mathcal{S} \supseteq \overline{\mathcal{E}_{3}(1)}
$$

4. Proof of the main result. We will prove our result in two parts according to the outline given in the previous section. First of all, we set up some notations. 
Notation 4.1. For a logical system $\mathcal{J}$ of inequalities which are combined by $\wedge$ and $\vee$, denote by $X(\mathcal{J})$ the set of all points that satisfy $\mathcal{J}$. Let $P$ be a set of inequalities. Then $\bigwedge P$ and $\bigvee P$ denote the systems $\bigwedge_{I \in P} I$ and $\bigvee_{I \in P} I$, respectively.

Table 1. The 43 cycles needed to cut out $\mathcal{D}_{3}^{0}$

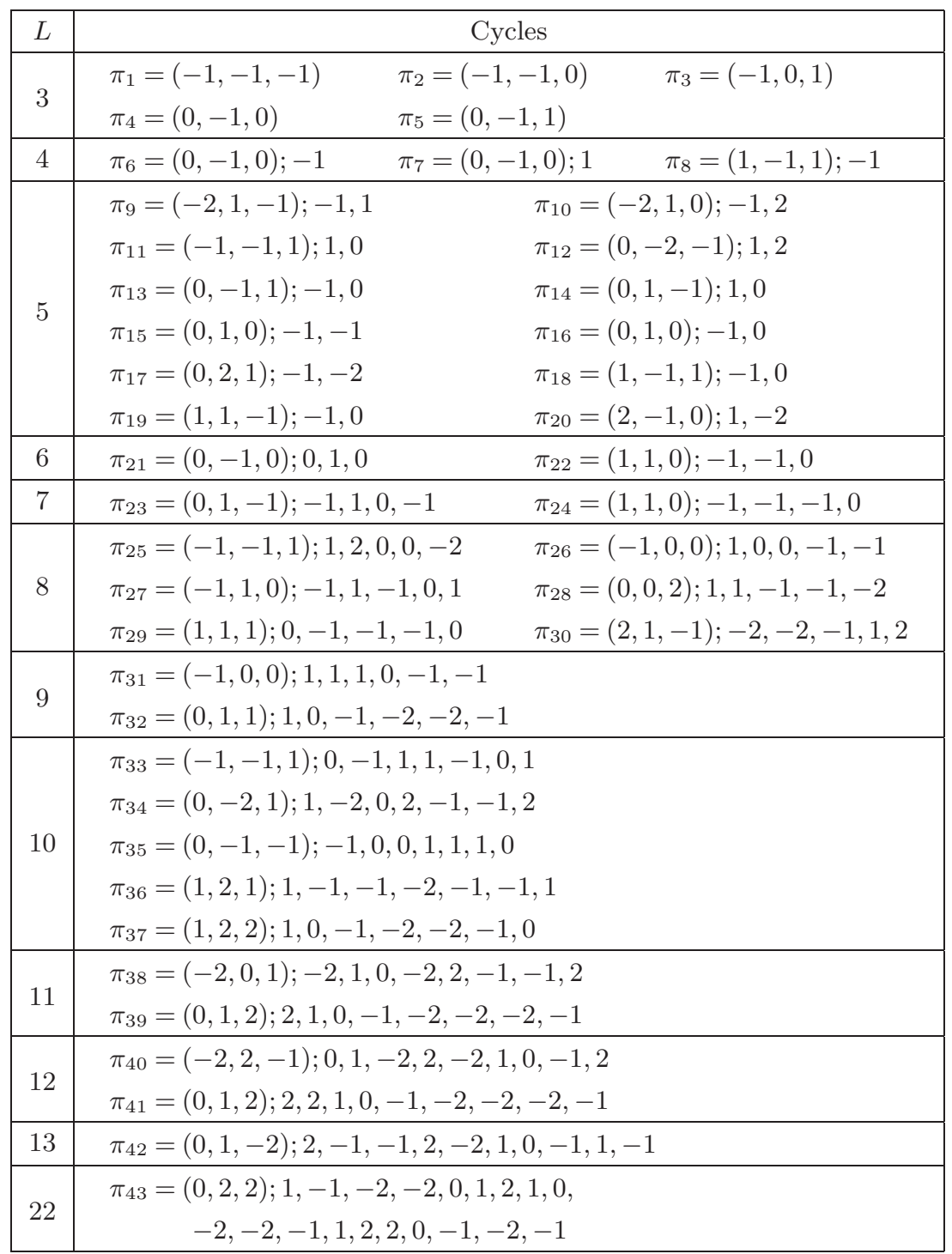

For the rest of the section denote by $T_{i}$ the set of inequalities that define the set $S_{i}$ for $i \in\{1, \ldots, 5\}$. These sets consist only of single inequalities. 
We have

$$
\begin{aligned}
T_{1}:= & \{2 x-2 z \geq 1,2 x+2 y+2 z>-1,2 x+2 y \leq 1,2 x \leq 1, \\
& 2 x-2 y+2 z \leq 1\} \\
T_{2}:=\{x-z \leq-1,2 x-2 y+2 z \leq 1,-2 x+2 y \leq 1,2 x>-1\} & \\
T_{3}:=\{x-z>-1,2 x-2 y+2 z \leq 1,-2 x+2 y<1,2 x>-1, & \\
& 2 x-2 z<-1,2 x+2 y+2 z>-1\} \\
T_{4}:=\{ & 2 x-2 y+2 z \leq 1,-2 x+2 y \leq 1,2 x-2 z \leq-1,2 x-2 z \geq-1, \\
& 2 x+2 y+2 z>-1\} \\
T_{5}:= & \{-1<2 x, 2 x \leq 1,-1<2 x-2 z, 2 x-2 z \leq 1,2 x+2 y+2 z>-1, \\
& 2 x-2 y+2 z \leq 1,2 x+4 y-2 z<3,2 y \leq 1\},
\end{aligned}
$$

hence the equality of $S_{4}$ and the two double inequalities of $S_{5}$ are split into single inequalities. Thus, $S_{i}=X\left(\bigwedge T_{i}\right)$ for $i=1, \ldots, 5$. Denote by $\bar{T}_{i}$ the set $T_{i}$ with all the strict inequalities changed to non-strict ones. Since all occurring inequalities are linear it can easily be checked that $\bar{S}_{i}=X\left(\bigwedge \bar{T}_{i}\right)$.

Table 1 shows 43 different cycles with corresponding period $L$; we denote the corresponding polyhedron by $P\left(\pi_{j}\right)$, where $j \in\{1, \ldots, 43\}$.

Now for each $i \in\{1, \ldots, 43\}$ define $Q_{i}$ as the set of single inequalities such that $P\left(\pi_{i}\right)=X\left(\bigwedge Q_{i}\right)$. For instance, the set $Q_{19}$ can be defined by

$$
\begin{aligned}
Q_{19}:=\{ & -1 / 2 \leq x+y-z-1, x+y-z-1<1 / 2,-1 / 2 \leq x-y-z, \\
& x-y-z<1 / 2,-1 / 2 \leq-x-y+1,-x-y+1<1 / 2, \\
& -1 / 2 \leq-x+z+1,-x+z+1<1 / 2,-1 / 2 \leq y+z-1, \\
& y+z-1<1 / 2\}
\end{aligned}
$$

(see also Example 2.5). Finally, we set

$$
\mathcal{P}:=\bigcup_{j=1}^{43} P\left(\pi_{j}\right) .
$$

REMARK 4.2. We note that the construction of the set $\mathcal{S}$ as well as the exhibition of the 43 cycles corresponding to relevant cutout polyhedra has been achieved by extensive computer experiments. Up to now we do not know an easy way that would lead to a list of all the cutouts needed to get the set $\mathcal{D}_{3}^{0}$. To find an algorithmic way to construct all these cutouts is desirable since it could lead to characterizations of $\mathcal{D}_{d}^{0}$ even for higher dimensions $d$.

Observe that no element of the 43 cycles given above contains elements having modulus greater than 2. Up to now, we do not know the reason for this fact. In order to characterize $\widetilde{\mathcal{D}}_{2}^{0}$ we need cycles with elements that are arbitrarily large (cf. [1, Sections 6 and 7]). 
4.1. Using the algorithm of Section 2. Theorem 2.3 shows the existence of an algorithm for the construction of a graph $G(H)=V \times E$ which can be used for finding all cycles of the mappings $\tau_{\mathbf{r}}$ with parameters $\mathbf{r}$ contained in the convex body $H$. Following [4], the graph is constructed recursively. Define $H=\square\left(\mathbf{r}_{1}, \ldots, \mathbf{r}_{k}\right) \subset \operatorname{int} \mathcal{D}_{3}$ to be the convex hull of some points $\mathbf{r}_{1}, \ldots, \mathbf{r}_{k}$. For $\mathbf{z} \in \mathbb{Z}^{d}$, let $m(\mathbf{z})=\min _{i \in\{1, \ldots, k\}}\left(-\left\lfloor\mathbf{r}_{i} \mathbf{z}\right\rfloor\right)$ and $M(\mathbf{z})=\max _{i \in\{1, \ldots, k\}}\left(-\left\lfloor\mathbf{r}_{i} \mathbf{z}\right\rfloor\right)$. Set

$$
V_{0}:=\left\{ \pm \mathbf{e}_{i} \mid i=1, \ldots, d\right\}
$$

and then successively calculate $V_{1}, V_{2}, \ldots$ by the rule

$$
V_{i+1}:=V_{i} \cup\left\{\left(z_{2}, \ldots, z_{d}, j\right) \mid \mathbf{z}=\left(z_{1}, \ldots, z_{d}\right) \in V_{i},-M(-\mathbf{z}) \leq j \leq M(\mathbf{z})\right\} .
$$

For sets $H$ having a sufficiently small diameter the iteration stabilizes, yielding $V:=V_{n}=V_{n+1}$ for some $n \in \mathbb{N}$. The set of edges is constructed by

$$
E:=\left\{\left(\mathbf{x},\left(z_{2}, \ldots, z_{d}, j\right)\right) \mid \mathbf{x}=\left(z_{1}, \ldots, z_{d}\right) \in V, m(\mathbf{z}) \leq j \leq M(\mathbf{z})\right\} .
$$

Let $\mathcal{Q}$ be a system of linear, non-strict inequalities linked with $\wedge$. Then $X(\mathcal{Q})$ forms a convex polyhedron that can be regarded as the convex hull of finitely many points $\mathbf{r}_{1}, \ldots, \mathbf{r}_{k}$. Suppose that $X(\mathcal{Q}) \subset \mathcal{E}_{3}(1)$. We want to set up an algorithm that calculates the set of all cycles $\pi$ whose associated polyhedron $P(\pi)$ has non-empty intersection with $X(\mathcal{Q})$. Theorem 2.3 ensures the existence of such an algorithm only if $X(\mathcal{Q})$ has sufficiently small diameter. If the set $X(\mathcal{Q})$ is too large, the graph $G(X(\mathcal{Q}))$ is infinite. We solve this problem in the following way. Suppose that, during the calculation of $|V|$, we obtain a set $V_{i}$ whose number of elements $\left|V_{i}\right|$ exceeds an appropriate bound $p$. In this case we stop the calculation of $V$ and divide $X(\mathcal{Q})$ into two parts for which we calculate the set $V$ again. By recursively doing this splitting procedure we eventually end up with sets whose diameter is small enough (provided that $p$ is chosen reasonably).

Suppose that $X(\mathcal{Q})$ is the convex hull of its $k$ vertices $\mathbf{r}_{1}, \ldots, \mathbf{r}_{k}$. We do not know these vertices explicitly. What we need is just $m(\mathbf{z})$ and $M(\mathbf{z})$ for certain fixed values of $\mathbf{z} \in \mathbb{Z}^{d}$. However, as $\mathcal{Q}$ is given as a system of linear inequalities, we easily see that

$$
m(\mathbf{z})=\min _{\mathbf{r} \in X(\mathcal{Q})}(-\lfloor\mathbf{r z}\rfloor), \quad M(\mathbf{z})=\max _{\mathbf{r} \in X(\mathcal{Q})}(-\lfloor\mathbf{r z}\rfloor) .
$$

The extremal values on the left hand side can now easily be calculated by standard linear optimization.

The algorithm consists of two parts. The first part, Algorithm 1, constructs the set of vertices $V$ of the graph $G(X(\mathcal{Q})$ ) for a given convex body $X(\mathcal{Q})$. Whenever during the calculation the size of this set exceeds a given bound $p$, Algorithm 1 stops, returning an overflow. Otherwise it terminates by returning $V$. Denote the application of Algorithm 1 with parameter $\mathcal{Q}$ and bound $p$ by $\operatorname{VG}(\mathcal{Q}, p)(\mathrm{VG}=$ vertices of the graph $)$. 


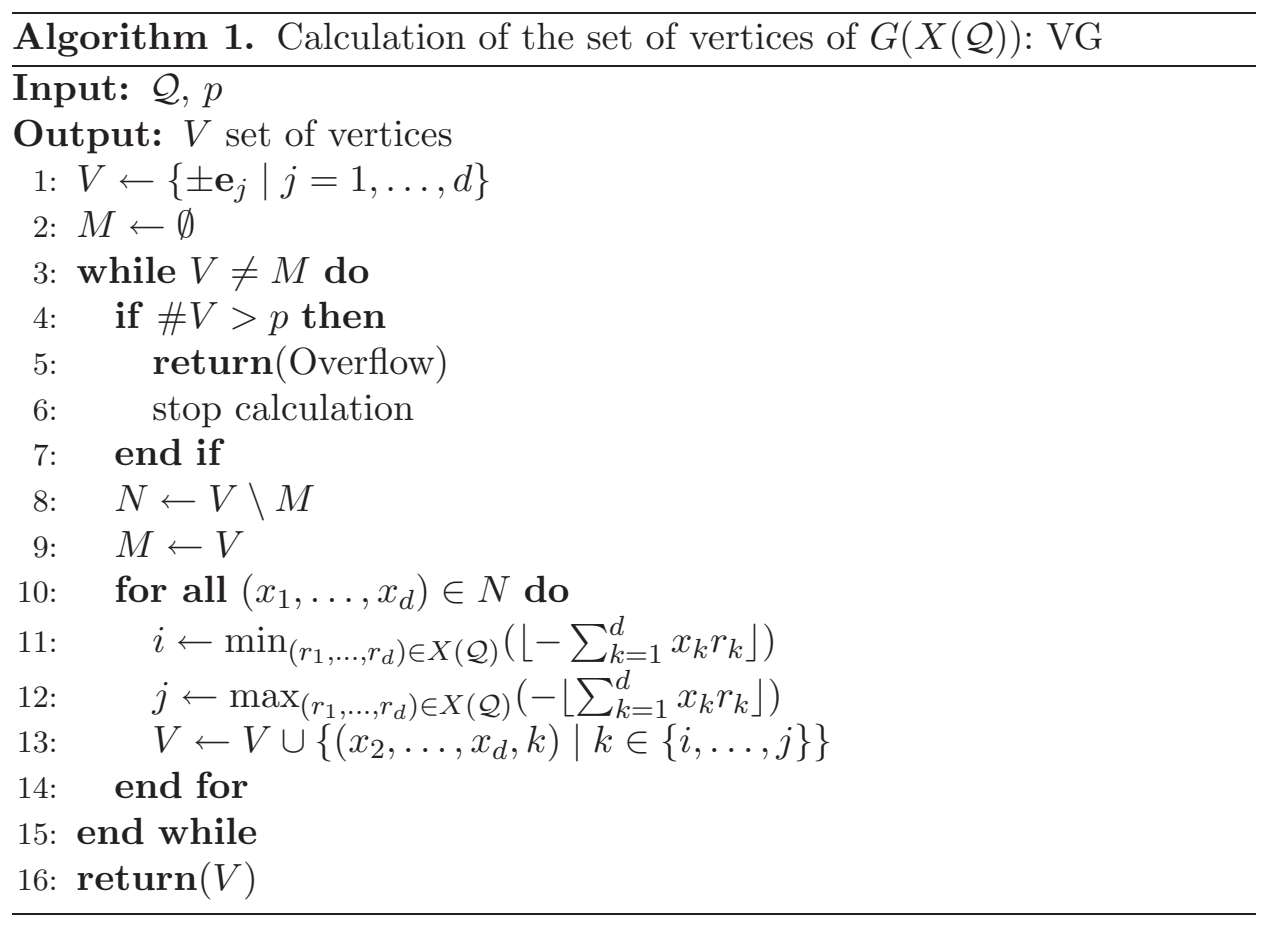

Algorithm 2 is recursive. As input we have $\mathcal{Q}$ and we write $\mathrm{FC}(\mathcal{Q})$ for its application on $\mathcal{Q}(\mathrm{FC}=$ find all cycles $)$. Algorithm 2 evokes Algorithm 1 to calculate the set of vertices of $G(X(\mathcal{Q})$ ). If an overflow occurs, the set $X(\mathcal{Q})$ is split with respect to some hyperplane $G\left(X_{1}, \ldots, X_{d}\right)=0$. Then Algorithm 2 is applied on $\mathcal{Q}_{1}:=\left(\mathcal{Q} \wedge G\left(X_{1}, \ldots, X_{d}\right) \leq 0\right)$ and $\mathcal{Q}_{2}:=$ $\left(\mathcal{Q} \wedge G\left(X_{1}, \ldots, X_{d}\right) \geq 0\right)$ separately. If there is no overflow and $V$ is returned, the set of edges $E$ is calculated and all the cycles of the graph are extracted. The cycles of the graph induce the cycles of $\tau_{\mathbf{r}}$ we are searching for. Note that the subsets $\mathcal{Q}_{1}$ and $\mathcal{Q}_{2}$ are again defined by finitely many non-strict inequalities so that they can be treated by Algorithm 1 in the same way as $\mathcal{Q}$.

In our setting we need to apply Algorithm 2 to the sets defined by the inequalities $\bar{T}_{i}(i \in\{1, \ldots, 5\})$. All we need to specify is the subdividing strategy and the bound $p$ for $|V|$. As for the subdividing strategy we subdivide a given set in two parts as follows. Let

$$
\begin{aligned}
& m_{i}:=\underset{\left(x_{1}, x_{2}, x_{3}\right) \in X(\mathcal{Q})}{\min } x_{i}, \quad i=1,2,3, \\
& M_{i}:=\max _{\left(x_{1}, x_{2}, x_{3}\right) \in X(\mathcal{Q})} x_{i}, \quad i=1,2,3,
\end{aligned}
$$

and $j \in\{1,2,3\}$ be the smallest index for which $M_{j}-m_{j}=\max \left(M_{1}-m_{1}\right.$, $\left.M_{2}-m_{2}, M_{3}-m_{3}\right)$. 


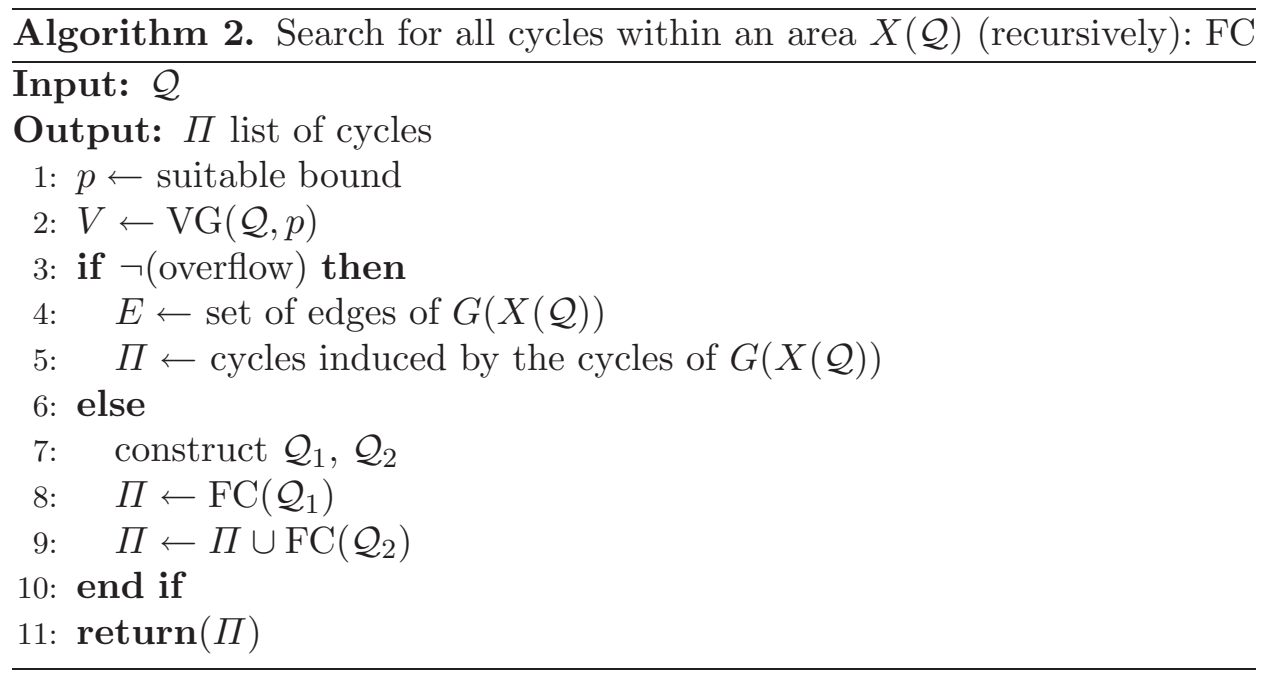

The dividing hyperplane is now defined by

$$
G\left(X_{1}, X_{2}, X_{3}\right)=0 \quad \text { with } \quad G\left(X_{1}, X_{2}, X_{3}\right):=X_{j}-\frac{M_{j}+m_{j}}{2} .
$$

For the upper bound of the number of vertices it turns out that a choice depending on the quantities $M_{j}-m_{j}$ is convenient. In particular, we choose $p=20 /\left(M_{j}-m_{j}\right)$. Then we get the following result:

LEMma 4.3. $\mathrm{FC}\left(\bigwedge T_{i}\right)$ terminates for each $i \in\{1, \ldots, 5\}$.

Proof. We implemented the algorithms for $T_{i}$ with the above mentioned subdivision strategy and bounds in Mathematica ${ }^{\circledR}$. The program is available on the authors' homepages [14].

Theorem 4.4. $S_{i} \subset \mathcal{D}_{3}^{0}$ for all $i \in\{1, \ldots, 5\}$.

Proof. For each $i \in\{1, \ldots, 5\}$ the set $X\left(\bigwedge \bar{T}_{i}\right)$ is the convex hull of finitely many points. Moreover, $X\left(\bigwedge \bar{T}_{i}\right)=\bar{S}_{i}$. Denote by $\Pi_{i}$ the set of cycles computed by the application of Algorithm 2 on $\bigwedge \bar{T}_{i}$. Hence $\Pi_{i}$ includes all cycles associated to polyhedra having non-empty intersection with $X\left(\bigwedge \bar{T}_{i}\right)$. Now, according to (2.2), each of these cycles $\pi \in \Pi_{i}$ induces a system of inequalities $\mathcal{P}(\pi)$. It turns out that for each $\pi \in \Pi_{i}$ we have

$$
X\left(\mathcal{P}(\pi) \wedge \bigwedge T_{i}\right)=\emptyset \quad \text { for each } i \in\{1, \ldots, 5\}
$$

(an easy way for checking this is to apply the cylindrical algebraic decomposition algorithm). Thus there is no cycle that yields a non-empty cutout intersecting $S_{i}$ and therefore $S_{i} \subset \mathcal{D}_{3}^{0}$.

4.2. Covering the set $\mathcal{D}_{3} \backslash \mathcal{D}_{3}^{0}$ by cutout polyhedra. Fix $Q_{1}, \ldots, Q_{43}$ to be the sets of inequalities of the 43 polyhedra induced by the cycles given 
in Table 1, where $Q_{j}$ denotes just the reduced set of inequalities such that $X\left(\bigwedge Q_{j}\right)$ yields the corresponding polyhedron for any $j \in\{1, \ldots, 43\}$. "Reduced" means that all the redundant inequalities are removed.

REMARK 4.5. It is not really necessary to work with the reduced systems but the main algorithm works much faster and the reduction is not too difficult to realize.

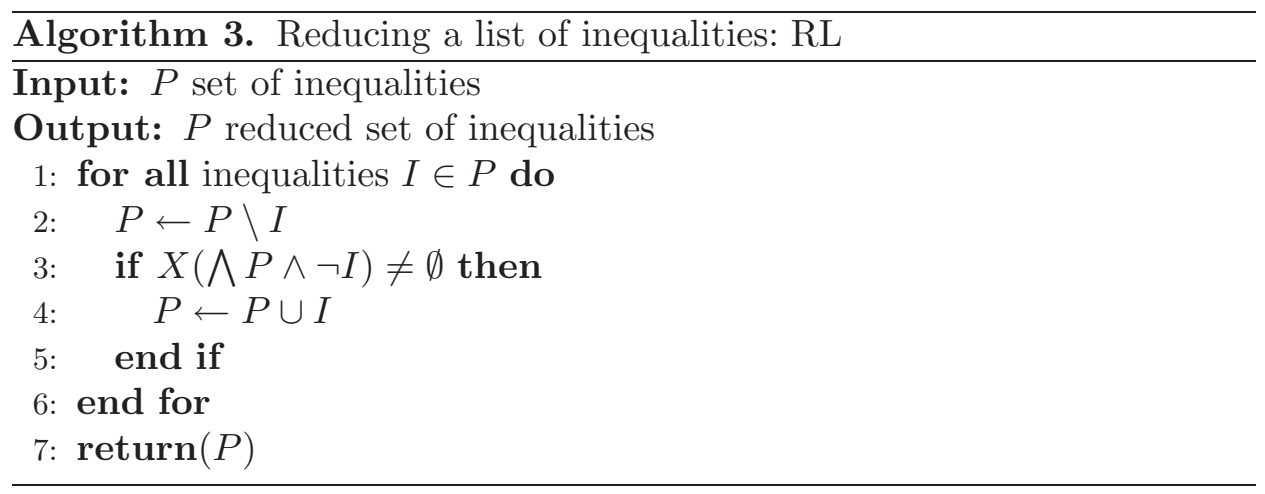

The algorithm simply uses the fact that an inequality $I$ is redundant for a system $\mathcal{S} \wedge I$ if $X(\mathcal{S} \wedge I)=X(\mathcal{S})$ or, equivalently, $X(\mathcal{S} \wedge \neg I)=\emptyset$. Denote the application of Algorithm 3 with parameter $P$ by $\mathrm{RL}(P)(\mathrm{RL}=$ reduce list of inequalities).

At the end of Section 2 we found a parametrization of $\overline{\mathcal{E}_{3}(1)}$. We saw that $\overline{\mathcal{E}_{3}(1)}=X(\bigwedge D)$ for

$$
D:=\left\{x+z \leq 1+y,-1-y \leq x+z, y-x z \leq 1-x^{2}, z \leq 3, z \geq-3\right\} .
$$

Let $\mathcal{P}$ be a list of sets of inequalities and $G$ be a set of inequalities. We want to verify if $\bigcup_{P \in \mathcal{P}} X(\bigwedge P)$ covers $X(\bigwedge G)$. This is equivalent to

$$
X\left(\bigwedge G \wedge \neg \bigvee_{P \in \mathcal{P}} \bigwedge P\right)=\emptyset
$$

In principle we could do this verification directly. For computational reasons we are a little more restricted. (In fact the direct verification of (4.1) overcharges Mathematica ${ }^{\circledR}$.) A verification of a claim of the shape (4.1) can be done in a reasonable amount of time if $\# \mathcal{P} \leq 3$. We give an algorithm that solves this problem for general $\mathcal{P}$ and $G$ by a subdivision process. The idea is to split the set $X(\bigwedge G)$ into suitable subsets and hope that each of these subsets is covered by a smaller number of sets. First we state Algorithm 4 which removes those sets from $\mathcal{P}$ that do not affect $G$, hence a set $P$ is removed when $X(\bigwedge G) \cap X(\bigwedge P)=\emptyset$. Denote the application of this algorithm by $\operatorname{RS}(G, \mathcal{P})(\mathrm{RS}=$ remove inequalities with respect to a set $)$. 
$\overline{\text { Algorithm 4. Removing those lists of inequalities that do not affect a given }}$ set $G$ : RS

Input: $G, \mathcal{P}$

Output: $\mathcal{P}$ reduced list of inequalities

1: for all sets $P \in \mathcal{P}$ do

2: $\quad$ if $X(\bigwedge G \wedge \bigwedge P)=\emptyset$ then

3: $\quad \mathcal{P} \leftarrow \mathcal{P} \backslash P$

4: $\quad$ end if

5: end for

6: $\operatorname{return}(\mathcal{P})$

The main algorithm (Algorithm 5 ) is recursive. As an input we have again $\mathcal{P}$ and $G$ of the usual shape, where $\mathcal{P}$ is reduced by Algorithm 4 . Whenever the algorithm recognizes that a subset of $X(\bigwedge G)$ is not fully covered by the sets described in $\mathcal{P}$, it returns this subset. Denote the application by $\mathrm{VC}(G, \mathcal{P})(\mathrm{VC}=$ verify covering $)$. At first Algorithm 5 checks whether $\# \mathcal{P} \leq 3$. If this is the case we can verify whether (4.1) holds, otherwise we choose an arbitrary inequality $I \in \bigcup_{P \in \mathcal{P}} P$ such that $X(\bigwedge G \wedge I) \neq X(\bigwedge G)$. There are two possibilities:

- There is such an inequality $I$. Then $X(\bigwedge G)$ is split by adding $I$ and $\neg I$, respectively, to $G$ and Algorithm 5 is applied (recursively) on both of these subsets. Algorithm 4 is used to possibly reduce $\mathcal{P}$ for each of the subsets. These reduced sets form the second parameter.

- There is no such $I$. But this would mean that all the points of $X(\bigwedge G)$ satisfy all inequalities of $\bigcup_{P \in \mathcal{P}} P$. This is equivalent to $X(\bigwedge G) \subset$ $X(P)$ for any $P \in \mathcal{P}$ and this implies that $G$ and $\mathcal{P}$ satisfy (4.1).

Now, whenever (4.1) is not fulfilled, the set $X(\bigwedge G)$ is not covered by $X\left(\bigvee_{P \in \mathcal{P}} \wedge P\right)$ and the algorithm returns the set $X(\bigwedge G)$. The application of Algorithm 5 terminates without any output if $X\left(\bigvee_{P \in \mathcal{P}} \wedge P\right)$ covers $X(\bigwedge G)$.

We can now state the main theorem of this subsection.

THEOREM 4.6. The algorithm $\operatorname{VC}(D, \mathcal{P})$ terminates without yielding any output for

$$
\mathcal{P}=\left\{Q_{1}, \ldots, Q_{43}, T_{1}, \ldots, T_{5}\right\} .
$$

Proof. We implemented the algorithms in Mathematica ${ }^{\circledR}$. The program is available on the authors' homepages [14].

Theorem 4.6 shows all the cutout polyhedra together with our set to really cover all of $\overline{\mathcal{E}_{3}(1)}$ and thus cover $\mathcal{D}_{3}$. More precisely, the cutout polyhedra $P\left(\pi_{1}\right), \ldots, P\left(\pi_{43}\right)$ cover the whole set $\overline{\mathcal{E}_{3}(1)} \backslash \mathcal{S}$. Hence, in view of 


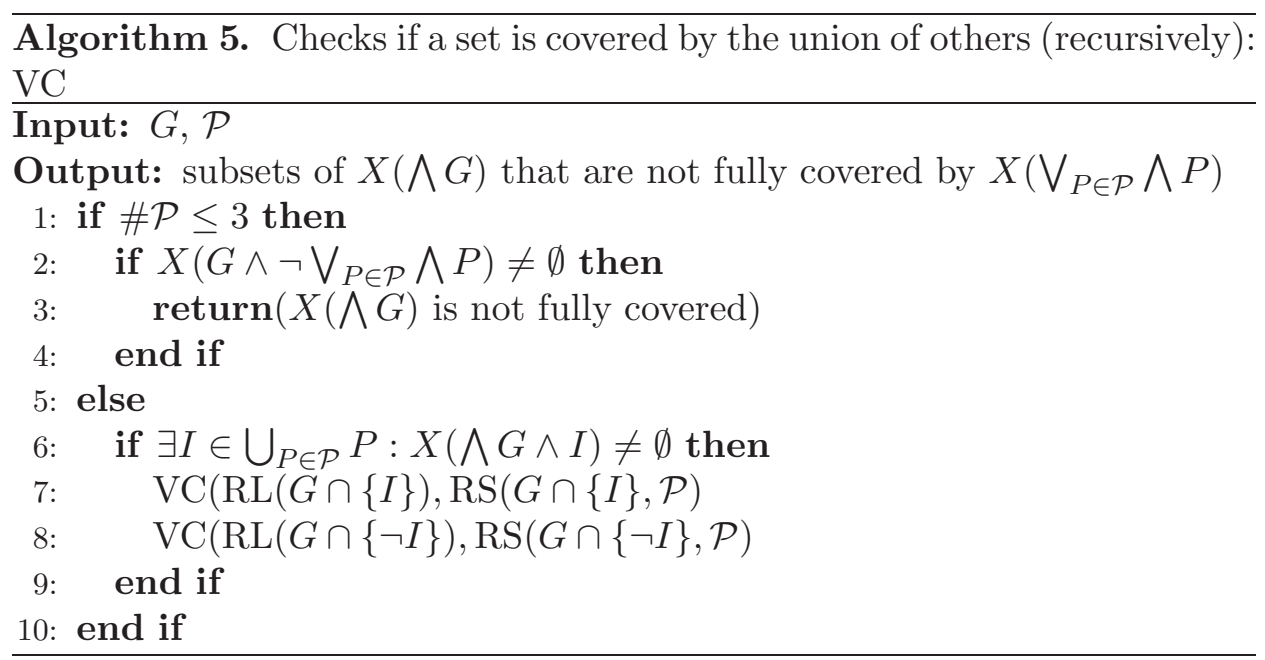

Theorem 4.4 we get

$$
\overline{\mathcal{E}_{3}(1)} \backslash \mathcal{S} \subset \bigcup_{1 \leq i \leq 43} P\left(\pi_{i}\right) .
$$

Together with Theorem 4.4 this yields Theorem 3.1 and we are done.

\section{References}

[1] S. Akiyama, T. Borbély, H. Brunotte, A. Pethő and J. M. Thuswaldner, Generalized radix representations and dynamical systems I, Acta Math. Hungar. 108 (2005), 207-238.

[2] S. Akiyama, H. Brunotte, A. Pethő and J. M. Thuswaldner, Generalized radix representations and dynamical systems II, Acta Arith. 121 (2006), 21-61.

[3] - - - - - - Generalized radix representations and dynamical systems III, Osaka J. Math., to appear.

[4] S. Akiyama and K. Scheicher, Symmetric shift radix systems and finite expansions, Math. Pannon. 18 (2007), 101-124.

[5] M. F. Barnsley, Fractals Everywhere, 2nd ed., Academic Press Professional, Boston, 1993.

[6] H. Brunotte, On trinomial bases of radix representations of algebraic integers, Acta Sci. Math. (Szeged) 67 (2001), 521-527.

[7] G. E. Collins, Quantifier elimination for the elementary theory of real closed fields by cylindrical algebraic decomposition, in: Lecture Notes in Comput. Sci. 33, Springer, 1975, 134-183.

[8] C. Frougny and B. Solomyak, Finite beta-expansions, Ergodic Theory Dynam. Systems 12 (1992), 713-723.

[9] W. Parry, On the $\beta$-expansions of real numbers, Acta Math. Acad. Sci. Hungar. 11 (1960), 401-416.

[10] A. Pethő, On a polynomial transformation and its application to the construction of a public key cryptosystem, in: Computational Number Theory, A. Pethö et al. (eds.), de Gruyter, 1991, 31-43. 
[11] A. Rényi, Representations for real numbers and their ergodic properties, Acta Math. Acad. Sci. Hungar. 8 (1957), 477-493.

[12] I. Schur, Über Potenzreihen, die im inneren des Einheitskreises beschränkt sind, J. Reine Angew. Math. 148 (1918), 122-145.

[13] P. Surer, New characterisation results for shift radix systems, submitted.

[14] —, Personal homepage, http://www.palovsky.com/links/p22007.htm.

[15] T. Takagi, Lectures in Algebra, Kyoritsu Publ., 1965.

Faculty of Informatics

University of Debrecen

P.O. Box 12

H-4010 Debrecen, Hungary

E-mail: husztia@inf.unideb.hu

Chair of Mathematics and Statistics

University of Leoben

Franz-Josef-Str. 18

A-8700 Leoben, Austria

E-mail: me@palovsky.com

joerg.thuswaldner@unileoben.ac.at
Johann Radon Institute for Computational and Applied Mathematics (RICAM)

Austrian Academy of Sciences

Altenbergerstr. 69

A-4040 Linz, Austria

E-mail: klaus.scheicher@oeaw.ac.at

Received on 24.8.2006

and in revised form on 26.6.2007 